\title{
Millicharged particles at electron colliders
}

\author{
Jinhan Liang, ${ }^{1}$ Zuowei Liu, ${ }^{1,2,3,4}$ Yue Ma, ${ }^{5}$ and Yu Zhang $\oplus^{6,7}$ \\ ${ }^{1}$ Department of Physics, Nanjing University, Nanjing 210093, China \\ ${ }^{2}$ Center for High Energy Physics, Peking University, Beijing 100871, China \\ ${ }^{3}$ Nanjing Proton Source Research and Design Center, Nanjing 210093, China \\ ${ }^{4}$ CAS Center for Excellence in Particle Physics, Beijing 100049, China \\ ${ }^{5}$ Kuang Yaming Honors School, Nanjing University, Nanjing 210023, China \\ ${ }^{6}$ Institutes of Physical Science and Information Technology, Anhui University, Hefei 230601, China \\ ${ }^{7}$ School of Physics and Materials Science, Anhui University, Hefei 230601, China
}

(Received 17 December 2019; accepted 9 June 2020; published 8 July 2020)

\begin{abstract}
We propose to search for millicharged particles in electron colliders operated with the center-of-mass energies at $\mathcal{O}(1-10) \mathrm{GeV}$, which include Belle II, BESIII, $B A B A R$, and also the proposed experiment Super Tau Charm Factory (STCF). We use the monophoton final state at electron colliders to probe the parameter space of millicharged particles, that is spanned by millicharge $\epsilon$ and mass $m$. We find that electron colliders have sensitivity to the previously unexplored parameter space for millicharged particles with MeV-GeV mass: $\epsilon \lesssim \mathcal{O}\left(10^{-1}\right)$ for $0.5 \mathrm{GeV} \lesssim m \lesssim 3.5 \mathrm{GeV}$ in $B A B A R, \epsilon \lesssim \mathcal{O}\left(10^{-3}\right)$ for $0.1 \mathrm{GeV} \lesssim$ $m \lesssim 1.5 \mathrm{GeV}$ in BESIII, $\epsilon \lesssim 10^{-3}-10^{-2}$ for $0.1 \mathrm{GeV} \lesssim m \lesssim 4 \mathrm{GeV}$ in Belle II, and $\epsilon \lesssim \mathcal{O}\left(10^{-4}\right)$ for $1 \mathrm{MeV} \lesssim m \lesssim 1 \mathrm{GeV}$ in STCF.
\end{abstract}

DOI: 10.1103/PhysRevD.102.015002

\section{INTRODUCTION}

Although anomaly cancellations link the electric charges of the standard model (SM) fermions [1], in principle, there is no such constraint for particles beyond the SM (BSM). For example, particles with arbitrarily small electric charge can naturally arise in models where hidden sector particles interact with the SM particles via kinetic mixing [2-4] or via Stueckelberg mixing [5-7]. A variety of experiments and theoretical investigations have been carried out to search for BSM particles with electric charge significantly smaller than the electron, which we refer to as millicharged particles (MCPs). The constraints on MCPs come both from terrestrial particle accelerators and from astrophysical processes. Previous particle accelerator constraints on MCPs include colliders [8-10], SLAC electron beam dump experiment [11], and E613 [12,13]. Recently, sensitivity of probing MCPs has also been studied in various accelerator experiments, including BESIII [14], LHC [15], Circular Electron Positron Collider (CEPC) [16], NA64 [17,18], and Light Dark Matter eXperiment (LDMX) [19]. Astrophysical constraints include white dwarf $[8,9,20]$, supernova $[9,21,22]$, cosmic microwave background (CMB) [23,24], big bang nucleosynthesis [8,9,25-27], red giants [8,20,25,26], and the

Published by the American Physical Society under the terms of the Creative Commons Attribution 4.0 International license. Further distribution of this work must maintain attribution to the author(s) and the published article's title, journal citation, and DOI. Funded by SCOAP ${ }^{3}$.
Sun [27]. MCPs can also be searched for in various neutrino experiments [28-32].

Recently, the $21 \mathrm{~cm}$ signal measured by the Experiment to Detect the Global Epoch of reionization Signature (EDGES) indicates that the Universe is colder than expected during the cosmic dawn [33]. Millicharged dark matter (DM) can provide cooling to cosmic hydrogen, leading to the strong $21 \mathrm{~cm}$ absorption signal [34-41].

In this paper, we study the experimental sensitivity on MCPs from electron colliders. The constraint on MCPs from the BESIII experiment has been recently studied in Ref. [14]; here, we extend the analysis to other electron colliders operated at the $\mathrm{GeV}$ scale, including Belle II, $B A B A R$, and also the proposed experiment, the Super Tau Charm Factory (STCF). Unlike the DM constraints which assume a sufficient amount of millicharged DM in our Universe, particle colliders can provide robust constraints on the MCPs which are independent on its composition in the Universe. At the $\mathrm{MeV}-\mathrm{GeV}$ scale, the leading constraints on MCPs come from colliders [8], SLAC [11], and Liquid Scintillator Neutrino Detector (LSND)/MiniBooNE [29]. We find that electron colliders can probe the previously unexplored MCP parameter space with $\mathrm{MeV}-\mathrm{GeV}$ mass. Our analysis also has a direct impact on millicharged DM models that are invoked to explain the $21 \mathrm{~cm}$ anomaly.

\section{ELECTRON COLLIDER SIGNALS}

In our analysis, we assume that the MCP is a Dirac fermion which is charged under the SM photon via the interaction Lagrangian $\mathcal{L}_{\text {int }}=e \varepsilon A_{\mu} \bar{\chi} \gamma^{\mu} \chi$, where $\chi$ is the 

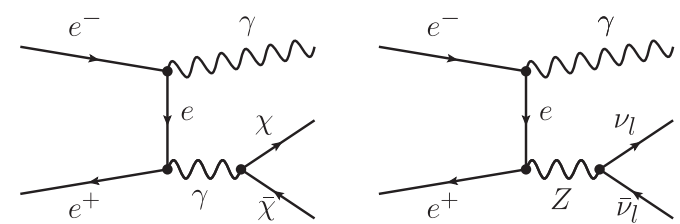

FIG. 1. Feynman diagrams for the process $e^{+} e^{-} \rightarrow \chi \bar{\chi} \gamma$ (left) and $e^{+} e^{-} \rightarrow \nu \bar{\nu} \gamma$ (right).

$\mathrm{MCP}$ and $A_{\mu}$ is the SM photon. The analysis presented here can be easily extended to MCPs with other spins.

Because the ionization signal of the MCP is typically undetectable in collider experiments, one thus relies on the visible final state particles produced in association with MCPs for the detection. Thus, we use the monophoton final state in electron colliders to search for MCPs [14]. The Feynman diagram for the signal process is shown in Fig. 1. The maximum photon energy is $E_{\gamma}^{\max }=\left(s-4 m_{\chi}^{2}\right) /(2 \sqrt{s})$, which applies to all detector cuts throughout this analysis. Here, $m_{\chi}$ is the MCP mass and $s$ is the square of center-ofmass energy.

Belle II is operated on SuperKEKB, which collides $7 \mathrm{GeV}$ electrons with $4 \mathrm{GeV}$ positrons [42]. SuperKEKB has a design luminosity of $8 \times 10^{35} \mathrm{~cm}^{-2} \mathrm{~s}^{-1}$ and expects to collect $50 \mathrm{ab}^{-1}$ integrated luminosity with 8 -yr data takings [42]. An upgrade with 5 times more luminosity is also anticipated with Belle II [43]. The BESIII detector is located at the Beijing Electron-Positron Collider II (BEPCII) with the beam energy ranging from 1.0 to $2.3 \mathrm{GeV}$ and luminosity of $10^{33} \mathrm{~cm}^{-2} \mathrm{~s}^{-1}$ [44]. STCF is a proposed experiment which collides electrons with positrons at the center-of-mass energies in the range 2-7 GeV, with the peak luminosity $\mathcal{O}\left(10^{35}\right) \mathrm{cm}^{-2} \mathrm{~s}^{-1}$ at $4 \mathrm{GeV}$ [45]. An integrated luminosity up to $20 \mathrm{ab}^{-1}$ is expected to be accumulated with a 10 -yr STCF running, assuming 9-month running time each year and $90 \%$ data taking efficiency [45]. The $B A B A R$ detector is operated at the PEP-II $e^{+} e^{-}$collider from 1999 to 2008 with most data collected near $\sqrt{s}=10.58 \mathrm{GeV}$ [the $\Upsilon(4 S)$ resonance] [46].

There are two types of monophoton background (BG): irreducible background and reducible background. The irreducible monophoton background is the SM final state containing one photon and two neutrinos; one of the irreducible background processes is shown in Fig. 1. The reducible monophoton background arises when a photon is produced in the final state together with several other visible particles which are, however, not detected due to the limitations of the detector acceptance. Belle II and $B A B A R$ have asymmetric detectors; BESIII and STCF have symmetric detectors. We discuss the reducible BG in detail later for each experiment, since it strongly depends on the angular coverage of the detectors.

\section{BELLE II}

In Belle II, photons and electrons can be detected in the electromagnetic calorimeter (ECL), which consists of three segments: a forward end cap with $12.4^{\circ}<\theta<31.4^{\circ}$, a barrel with $32.2^{\circ}<\theta<128.7^{\circ}$, and a backward end cap with $130.7^{\circ}<\theta<155.1^{\circ}$ in the lab frame [42]. There are two important monophoton reducible backgrounds [42]: One is mainly due to the lack of polar angle coverage of the ECL near the beam directions, which is referred to as the "bBG"; the other one is mainly due to the gaps between the three segments in the ECL detector, which is referred to as the "gBG."

In the bBG, all the final state particles except the detected monophoton are emitted with $\theta>155.1^{\circ}$ or $\theta<12.4^{\circ}$ in the lab frame. Some major bBG processes include $e^{+} e^{-} \rightarrow$ $\downarrow \gamma \gamma$ and $e^{+} e^{-} \rightarrow \ell^{+} \ell^{-} \gamma$, where $\ell=e, \mu, \tau$; the final state particles with a slash on the name are emitted along the beam directions.

For symmetric detectors, such as BESIII and STCF, the maximum energy of the monophoton events in the bBG in the c.m. frame, $E_{\gamma}^{m}$, is given by

$$
E_{\gamma}^{m}\left(\theta_{\gamma}\right)=\sqrt{s}\left(1+\frac{\sin \theta_{\gamma}}{\sin \theta_{b}}\right)^{-1},
$$

where $\theta_{b}$ is the polar angle corresponding to the edge of the detector [16]. For the Belle II detector, which is asymmetric, $E_{\gamma}^{m}$ in the c.m. frame is given by (if not exceeding $\sqrt{s} / 2)$

$$
E_{\gamma}^{m}\left(\theta_{\gamma}\right)=\frac{\sqrt{s}\left(A \cos \theta_{1}-\sin \theta_{1}\right)}{A\left(\cos \theta_{1}-\cos \theta_{\gamma}\right)-\left(\sin \theta_{\gamma}+\sin \theta_{1}\right)}
$$

with the maximum of where all angles are given in the c.m. frame, and $A=\left(\sin \theta_{1}-\sin \theta_{2}\right) /\left(\cos \theta_{1}-\cos \theta_{2}\right)$, with $\theta_{1}$ and $\theta_{2}$ being the polar angles corresponding to the edges of the ECL detector. ${ }^{1}$ To remove the above $\mathrm{bBG}$, the detector cut $E_{\gamma}>E_{\gamma}^{m}$ is used (hereafter the bBG cut), which is shown in Fig. 2.

Because the ECL gaps are significantly away from the beam direction, the monophoton energy of the $\mathrm{gBG}$ can be quite large in the central $\theta_{\gamma}$ region. The gBG simulations have been carried out by Ref. [42] in searching for an invisibly decaying vector boson. The dominated $\mathrm{gBG}$ is $e^{+} e^{-} \rightarrow \gamma \gamma \gamma(\gamma)$ with at least one final state photon emitting through the gaps [42]. For example, one major background arises when one final state photon in the process $e^{+} e^{-} \rightarrow$ $\gamma \gamma \gamma$ escapes via the gap between the ECL barrel and the backward end cap, and the second photon is emitted along the beam direction [47]. Two different sets of detector cuts are designed by Ref. [42] to optimize the detection efficiency for different masses of the vector boson, which are shown in Fig. 2. The low-mass region in the monophoton phase space has few gBG events, which is applied

\footnotetext{
${ }^{1}$ The polar angle in the c.m. frame is related to that in the lab frame via $\tan \theta_{\mathrm{c.m} .}=\sin \theta_{\text {lab }} /\left(\gamma \cos \theta_{\text {lab }}-\gamma \beta\right)$, where $\beta=3 / 11$ and $\gamma=1 / \sqrt{1-\beta^{2}}$.
} 


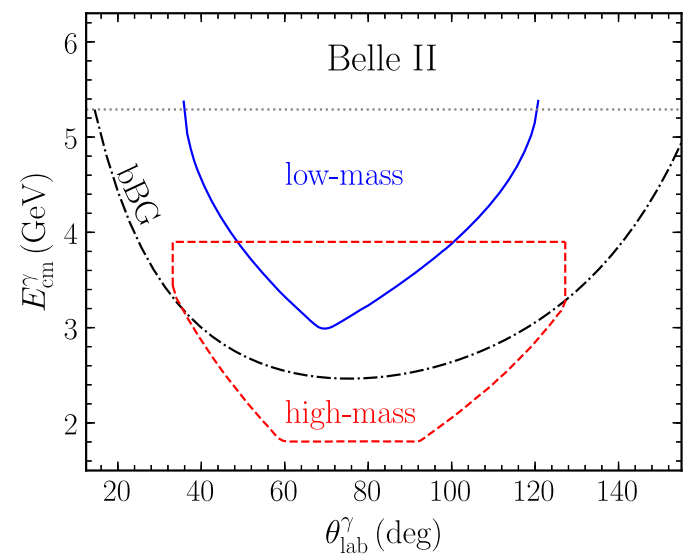

FIG. 2. Monophoton phase space $E_{\mathrm{c} . \mathrm{m} .}^{\gamma}-\theta_{\mathrm{lab}}^{\gamma}$ in Belle-II. $E_{\mathrm{c} . \mathrm{m}}^{\gamma}$ is the photon energy in the c.m. frame; $\theta_{\text {lab }}^{\gamma}$ is the photon polar angle with respect to the initial electron in the lab frame. We refer to the region above the blue solid line as the "low-mass" region and the region enclosed by the red dashed line as the "high-mass" region. Both the low-mass and high-mass regions are taken from simulations given in Ref. [42]. The gray dotted line indicates the $\sqrt{s} / 2$ value in Belle-II. The black dot-dashed line is the bBG cut.

for the vector boson with mass less than $6 \mathrm{GeV}$. However, if the vector boson mass is in the range $6-8 \mathrm{GeV}$, only lowenergy photons can be produced in the new physics processes so that the high-mass cut region is preferred.

To probe the millicharge, we define $\chi^{2}(\epsilon) \equiv S^{2} /(S+B)$ [48], where $S(B)$ is the number of events in the signal (background) processes. The 95\% confidence level (C.L.) upper bound on the millicharge, $\epsilon_{95}$, is obtained by solving $\chi^{2}\left(\epsilon_{95}\right)-\chi^{2}(0)=2.71$. Figure 3 shows the expected 95\% C.L. upper bound on millicharge using the low-mass and high-mass cuts with $50 \mathrm{ab}^{-1}$ data. We calculate the signal and irreducible background events by integrating the

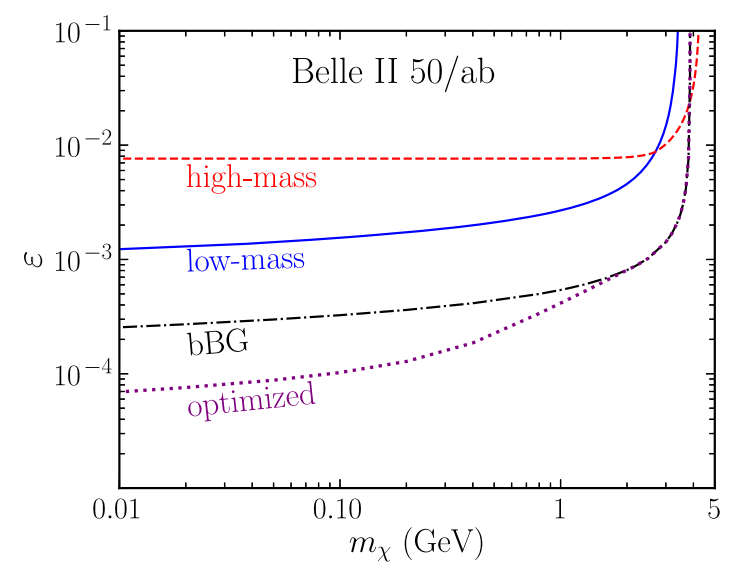

FIG. 3. The expected $95 \%$ confidence level (C.L.) upper bound on millicharge at Belle II under the low-mass cut (solid line) and the high-mass cut (dashed line), with $50 \mathrm{ab}^{-1}$ integrated luminosity. The black (purple) line corresponds to the limit using the bBG (optimized) cut. differential monophoton cross sections in different regions of the phase space under different detector cuts and assuming photon detection efficiency as 95\% [42]. Our calculation shows that there are about $10900(2280,15230)$ irreducible BG events with the bBG (low-mass, high-mass) cut with $50 \mathrm{ab}^{-1}$ integrated luminosity. For the reducible background, it is found that about 300 (25000) gBG events survived the low-mass (high-mass) cuts with $20 \mathrm{fb}^{-1}$ integrated luminosity [42], which are rescaled according to the luminosity. The constraint with the high-mass cut becomes better than the low-mass cut when the MCP mass exceeds $\sim 3 \mathrm{GeV}$.

We also compute the limits without taking gBG into account, in order to compare with other experiments where detailed simulations with $\mathrm{gBG}$ are not available. We use the bBG cut to remove the reducible background events; the $B G$ events that survived the $\mathrm{bBG}$ cut are due to irreducible backgrounds, if $\mathrm{gBG}$ is not considered. We integrated the monophoton differential cross section for MCPs [14] and for SM irreducible BG [14], with the bBG cut to obtain the number of events. The 95\% C.L. upper bound analyzed with the bBG cut is shown in Fig. 3, where gBG is not considered; the upper bound is about 5 times stronger than the one when $\mathrm{gBG}$ is considered under the low-mass cut, for millicharged particles with mass less than $1 \mathrm{GeV}$.

\section{BESIII AND STCF}

It has been recently proposed to search for MCPs in BESIII [14]. Here we update the BESIII sensitivity by taking into account the most recent data: $1.4(0.13,0.5) \mathrm{fb}^{-1}$ at $\sqrt{s}=3.097(3.554,3.686) \mathrm{GeV}$ [49]. In BESIII, we have $\cos \theta_{b}=0.95$, taking into account the coverage of main drift chamber (MDC), electromagnetic calorimeter (EMC), and time-of-flight (TOF) [14]. We adopt the detector cuts for photons by the BESIII Collaboration (hereafter the preselection cuts) [50]: $E_{\gamma}>25 \mathrm{MeV}$ with $|\cos \theta|<0.8$ or $E_{\gamma}>$ $50 \mathrm{MeV}$ with $0.86<|\cos \theta|<0.92$. We further apply the $\mathrm{bBG}$ cut to remove the reducible background. We compute the number of events under both preselection cuts and the bBG cut given in Eq. (1). We define $\chi_{\text {tot }}^{2}(\epsilon)=\sum_{i} \chi_{i}^{2}(\epsilon)$, where $\chi_{i}^{2}(\epsilon) \equiv S_{i}^{2} /\left(S_{i}+B_{i}\right)$ for each BESIII colliding energy. The $95 \%$ C.L. upper bound on millicharge from BESIII is obtained by demanding $\chi_{\text {tot }}^{2}\left(\epsilon_{95}\right)=\chi^{2}(0)+2.71$, which is shown in Fig. 8.

A total luminosity of $20 \mathrm{ab}^{-1}$ is expected at the future STCF experiment operated at $\sqrt{s}=2-7 \mathrm{GeV}$. Although the STCF luminosity is a little smaller than Belle II, the smaller colliding energy in STCF enhances the sensitivity to sub-GeV MCPs. Figure 4 shows the monophoton cross section in the new physics model and in irreducible BG; the signal to background ratio increases when the colliding energy decreases. STCF is thus the ideal experiment to search for light MCPs because of both the high integrated luminosity and the relatively low colliding energy. 


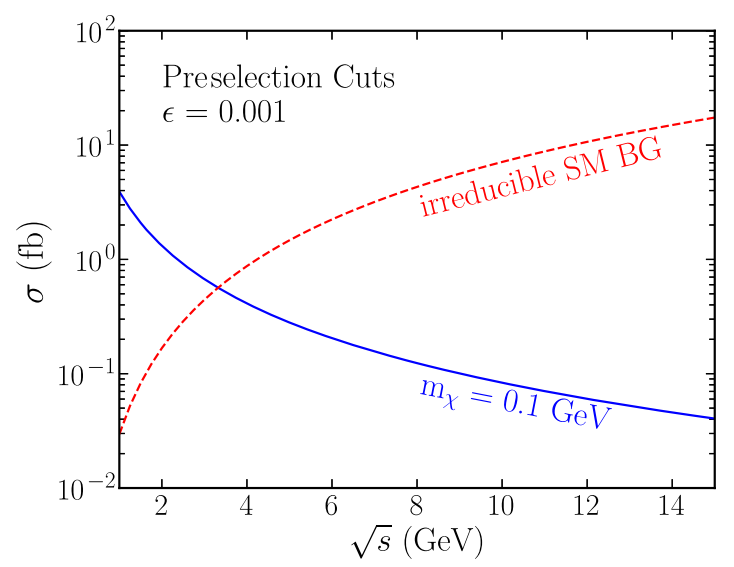

FIG. 4. Monophoton cross section as a function of $\sqrt{s}$ for MCPs (solid line) and for irreducible BG (dashed line). Only preselection cuts are applied. We use $\epsilon=0.001$ and $m_{\chi}=$ $0.1 \mathrm{GeV}$ for the MCPs.

We use the BESIII detector parameters to analyze the constraints from STCF because of the similarity of the two experiments. Figure 5 shows the expected STCF limits on millicharge assuming $10 \mathrm{ab}^{-1}$ luminosity at three different colliding energies. We compute the signal and irreducible background under both the preselection cuts and the bBG cut; the irreducible BG yields about $27 \mathrm{pb}$ at $\sqrt{s}=$ $4 \mathrm{GeV}$ under these cuts. The STCF can probe $\epsilon \simeq 10^{-4}$ for mass around $10 \mathrm{MeV}$, if operated at $\sqrt{s}=2 \mathrm{GeV}$ with $10 \mathrm{ab}^{-1}$ data.

To our knowledge, BESIII has not released any analysis on gBG. Thus, we neglect gBG in the BESIII and STCF analyses. Improved BESIII and STCF limits can be obtained in the future when the $\mathrm{gBG}$ analysis is available.

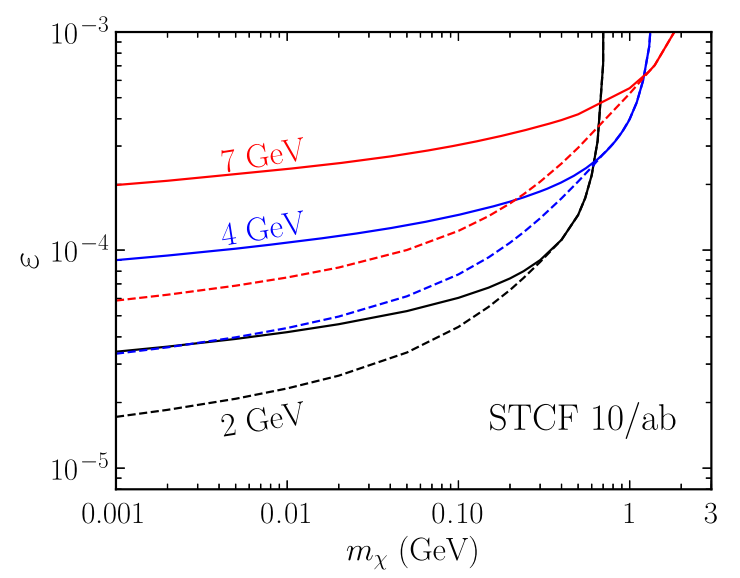

FIG. 5. The expected 95\% C.L. exclusion limits on millicharge at STCF at various colliding energies with $10 \mathrm{ab}^{-1}$ luminosity. Solid (dashed) curves indicate the limits under the bBG (optimized) cut for $\sqrt{s}=7,4,2 \mathrm{GeV}$ in descending order.

\section{BABAR}

To probe MCPs, we use the monophoton events collected by the BABAR Collaboration [51] which were previously analyzed to search for the light scalar particle $A^{0}$ produced via $e^{+} e^{-} \rightarrow \Upsilon(3 S) \rightarrow \gamma A^{0}$; two sets of data are analyzed in Ref. [51]: the $28 \mathrm{fb}^{-1}$ "high-E" photons with $3.2 \mathrm{GeV}<E_{\text {c.m. }}^{\gamma}<5.5 \mathrm{GeV}, \quad-0.31<$ $\cos \left(\theta_{\mathrm{c} \text {.m. }}^{\gamma}\right)<0.6$, and $\cos \left(6 \phi_{\mathrm{c} . \mathrm{m} \text {. }}^{\gamma}\right)<0.96$ corresponding to the instrumented flux return fiducial; the $19 \mathrm{fb}^{-1}$ "low-E" photons with $2.2 \mathrm{GeV}<E_{\mathrm{c} . \mathrm{m} .}^{\gamma}<3.7 \mathrm{GeV}$ and $-0.46<$ $\cos \left(\theta_{\text {c.m. }}^{\gamma}\right)<0.46$.

The detector cuts used in the BABAR analysis [51] can be divided into two categories: geometric cuts and nongeometric cuts; we compute the detector efficiency separately for these two cuts, following Ref. [52].

The detector efficiency for geometric cuts in the high-E (low-E) region is about $34 \%$ (37\%) for the $\left(1+\cos ^{2} \theta_{\gamma}\right)$ angular distribution used in Ref. [51].

Because the total detector efficiency for $e^{+} e^{-} \rightarrow$ $\Upsilon(3 S) \rightarrow \gamma A^{0}$ is $(10-11) \%$ (20\%) in the high-E (low-E) region [51], the detector efficiency for the nongeometric cuts (denoted as $\mathrm{f}_{\mathrm{NG}}$ ) is about $30 \%(54 \%)$ in the high-E (low-E) region. The signal events under the high-E and low-E detector cuts is computed via

$N_{s}=\mathcal{L} f_{\mathrm{NG}} \int d \Omega d E_{\gamma}^{d} d E_{\gamma} f\left(E_{\gamma}^{d}, E_{\gamma}, \sigma\left(E_{\gamma}\right)\right) \frac{d \sigma}{d E_{\gamma} d z_{\gamma}}$,

where $d \sigma /\left(d E_{\gamma} d z_{\gamma}\right)$ is the differential cross section [14], $z_{\gamma}=\cos \left(\theta_{\gamma}\right), E_{\gamma}^{d}$ is the detected photon energy, and $\mathcal{L}=$ $28(19) \mathrm{fb}^{-1}$ for high-E (low-E) data [46,51]. Here the photon energy is smeared via the crystal ball function $f\left(E_{\gamma}^{d}, E_{\gamma}, \sigma\left(E_{\gamma}\right)\right)$ with the energy resolution $\sigma\left(E_{\gamma}\right) / E_{\gamma}=$ $1.5 \%\left(E_{\gamma} / \mathrm{GeV}\right)^{1 / 4} \oplus 1 \%[52]$.

Following Refs. [51,52], we model the background using fitting functions: We use a crystal ball function peaked at $m_{\chi \chi}=0$, where $m_{\chi \chi}^{2}=s-2 \sqrt{s} E_{\gamma}$, with normalization $N_{1}$ and $N_{2} \exp \left(c m_{\chi \chi}^{2}\right)$ for the high-E region; we use $N_{3} \exp \left(c_{1} m_{\chi \chi}^{2}+c_{2} m_{\chi \chi}^{4}\right)$ and a constant term $N_{4}$ for the low-E region. The 95\% C.L. upper bound on millicharge is computed using the profile likelihood method. The likelihood function we use is

$$
\mathcal{L}=\max \left\{\prod_{i=1}^{\text {bins }} \exp \left[\frac{\left(N_{s}^{i}+N_{b}^{i}-N_{o}^{i}\right)^{2}}{2 \sigma_{i}^{2}}\right]\right\}
$$

where $N_{s}^{i}\left(N_{b}^{i}, N_{o}^{i}\right)$ is the number of signal (background, observed) events in bin $i$ and $\sigma_{i}$ is the error bar. We use $N_{1}$, $N_{2}, N_{3}, c, c_{1}$, and $c_{2}$ as nuisance parameters. The upper bound on millicharge from the BABAR data is shown in Fig. 6. 


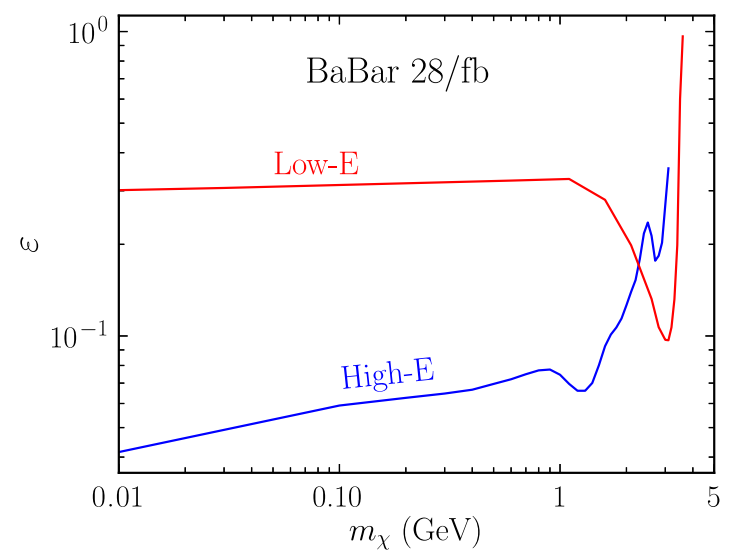

FIG. 6. The 95\% limits on $\epsilon$ with the profile likelihood method at $B A B A R$. The blue (red) solid line shows the limit with $B A B A R$ high-E (low-E) data.

\section{OPTIMIZED CUT}

We further carry out a preliminary analysis in which we optimize the detector cuts by considering the irreducible background only. For the monophoton process at the low-energy electron collider, the irreducible background decreases with the photon energy; the monophoton cross section in the MCP models, however, is relatively larger in the high-energy region than the low-energy region.

Thus, selecting photons with relatively high energy can enhance discovery sensitivity. To find the optimized cut, in addition to the bBG cut, we select photons in the range $E_{\gamma}^{\min }<E_{\gamma}<E_{\gamma}^{\max }$, where $E_{\gamma}^{\max }=\left(s-4 m_{\chi}^{2}\right) /(2 \sqrt{s})$, and vary $E_{\gamma}^{\min }$ to find the best limit on millicharge. Furthermore, the energy difference $\Delta E_{\gamma} \equiv E_{\gamma}^{\max }-E_{\gamma}^{\min }$ is required to be larger than the photon energy resolution $\sigma_{E}$, when $E_{\gamma}^{\max }$ is

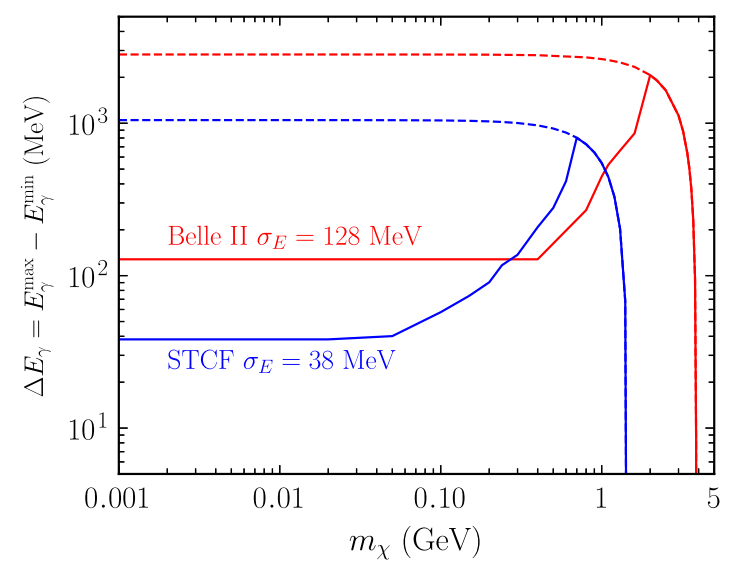

FIG. 7. The $\Delta E_{\gamma}$ value that yields the best limit on millicharge in Belle II and STCF. We use 50(20) $\mathrm{ab}^{-1}$ data here for Belle II (STCF). We consider $\sqrt{s}=4 \mathrm{GeV}$ for STCF. The dashed lines indicate the bBG cuts. more than $1 \sigma_{E}$ above the minimum value of the bBG cut curve.

Figure 7 shows the $\Delta E_{\gamma}$ that gives rise to the best limit on millicharge in Belle II and STCF. For STCF, we use the photon resolution of the EMC in BESIII $\sigma_{E} / E=$ $2.3 \% / \sqrt{E / \mathrm{GeV}} \oplus 1 \%$ [44], and we take $\sigma_{E}=38 \mathrm{MeV}$ for light mass. For Belle II, $\sigma_{E} / E=4 \%(1.6 \%)$ at 0.1 (8) $\mathrm{GeV}$ [42], and we take $\sigma_{E}=128 \mathrm{MeV}$ for light mass. As shown in Fig. 7, the best $\Delta E_{\gamma}$ value is equal to the photon energy resolution for light mass. For high mass, because $E_{\gamma}^{\max }$ starts to approach the bBG cut, $\Delta E_{\gamma}$ can become smaller than the photon energy resolution.

\section{RESULTS}

Figure 8 summarizes the sensitivity on millicharge $\epsilon$ from the low-energy electron colliders, including Belle II, STCF, BESIII, and BABAR. The BABAR and Belle II limits,

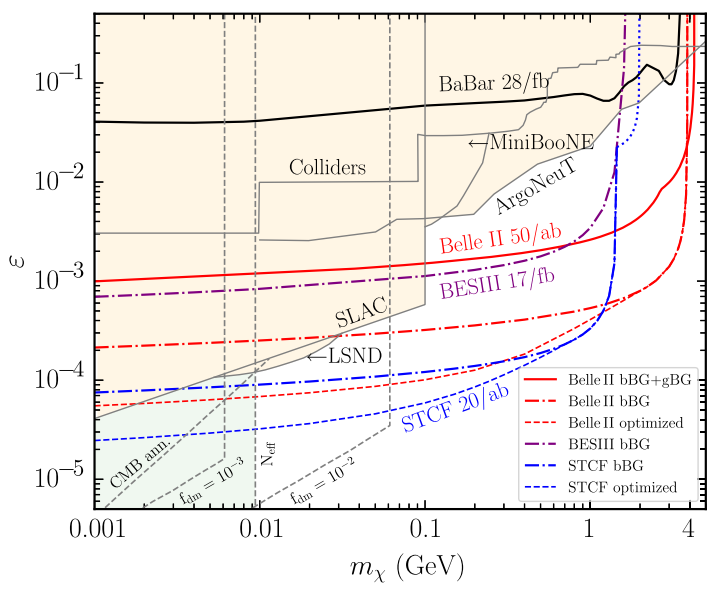

FIG. 8. The expected $95 \%$ C.L. exclusion limits on MCPs at Belle II, BESIII, STCF, and BABAR. The BABAR limit (black solid line) is obtained by combining the high-E limit and low-E limit in Fig. 6. The Belle II limit (red solid line) combines the low-mass and high-mass limits in Fig. 3, where both the bBG and the $\mathrm{gBG}$ are considered. The other two Belle II limits (red dotdashed and red dashed lines) are obtained with the (bBG and optimized) cuts where the gBG is omitted. The BESIII limit (purple dot-dashed line) is obtained with the integrated luminosity during 2011-2018 where the gBG is omitted. The STCF limits (blue dot-dashed and blue dashed lines) are obtained for $\sqrt{s}=$ $4 \mathrm{GeV}$ and $20 \mathrm{ab}^{-1}$ under the (bBG and optimized) cuts, where the $\mathrm{gBG}$ is omitted. Beyond mass $\sim 1.4 \mathrm{GeV}$, we analyze the monophoton signal using the preselection cuts to obtain the STCF limit, which is shown as the blue dotted curve. Constraints from colliders [9,15], SLAC [11], LSND [29], MiniBooNE [29], and ArgoNeuT [53] are also presented. The parameter space of millicharged DM to explain the EDGES $21 \mathrm{~cm}$ anomaly [33] is also shown where $f_{\mathrm{dm}}=10^{-3}\left(10^{-2}\right)$ is the millicharged DM fraction [34]. The excluded regions from cosmology are also shown, including the bounds from $\mathrm{N}_{\text {eff }}[36,54]$ and the dark matter annihilation impact on CMB with $f_{\mathrm{dm}}=10^{-2}[36,55]$. 
shown as solid curves in Fig. 8, have been analyzed taking into account the various SM backgrounds. With existing data from $B A B A R$, the previously allowed parameter space with millicharge $\epsilon \sim 10^{-1}$ and mass $\sim(1-3) \mathrm{GeV}$ can be probed. Because of the higher luminosity expected at Belle II, a larger parameter space that is previously unconstrained by other experiments is going to be explored by Belle II; with $50 \mathrm{ab}^{-1}$ data, millicharge down to $\sim 10^{-2}-10^{-3}$ for mass $\sim(0.1-4) \mathrm{GeV}$ is expected to be probed by Belle II.

The STCF and BESIII limits, shown as dot-dashed curves in Fig. 8, are obtained when the background due to the gaps in the detectors is neglected. BESIII can probe new parameter space for mass $>100 \mathrm{MeV}$, with $17 \mathrm{fb}^{-1}$ data collected during 2011-2018. The future STCF can probe millicharge parameter space below the SLAC experiment [11]. With $20 \mathrm{ab}^{-1}$ data at $\sqrt{s}=4 \mathrm{GeV}$, STCF can provide leading constraints on millicharge, $\epsilon \lesssim \mathcal{O}\left(10^{-4}\right)$ for mass from $3 \mathrm{MeV}$ to about $1 \mathrm{GeV}$. The expected limit from STCF also eliminates some regions of the MCP parameter space where the $21 \mathrm{~cm}$ anomaly could be explained due to cooling from millicharged DM.

In addition to the initial state radiation process in Fig. 1, the $\chi \chi \gamma$ final state can also occur in meson decays, which can improve the sensitivity for the low-mass region. However, this is beyond the scope of this work. Under the bBG cut, STCF loses sensitivity to MCPs when $m_{\chi} \gtrsim 1.5 \mathrm{GeV}$, since $E_{\gamma}^{\max }=\left(s-4 m_{\chi}^{2}\right) /(2 \sqrt{s})$ is now lower than the minimum energy of the bBG cut. To estimate the STCF sensitivity for $m_{\chi} \gtrsim 1.5 \mathrm{GeV}$, we apply only the preselection cuts; the dominant $\mathrm{BG}$ now is due to the $e^{+} e^{-} \rightarrow \phi^{+} \phi^{-} \gamma$ process. The STCF limit in the highmass region is shown as the blue dotted curve in Fig. 8.

The omission of the $\mathrm{gBG}$ in BESIII $\left(17 \mathrm{fb}^{-1}\right)$ leads to a stronger limit than Belle II $\left(50 \mathrm{ab}^{-1}\right)$ with $\mathrm{gBG}$ included for $m \lesssim 0.7 \mathrm{GeV}$. To compare the capability of probing the parameter space from different experiments, we also present a Belle II limit (dot-dashed curve) with gBG omitted. Although the STCF luminosity is lower than Belle II, STCF has better sensitivity in probing the lowmass region $(m \lesssim 1 \mathrm{GeV})$ than Belle II. This is because STCF is operated at a lower colliding energy where the monophoton cross section in MCPs (SM) is larger (smaller) than Belle II. The one order of magnitude difference in sensitivity between the two Belle II limits, the solid curve and the dot-dashed curve in Fig. 8, shows that the control on $\mathrm{gBG}$ is very important in probing the MCP parameter space. Since the dot-dashed curves in Fig. 8 are obtained without $\mathrm{gBG}$, the actual limits should be weaker when $\mathrm{gBG}$ is taken into account. However, if the reducible background due to gaps in the detector can be significantly suppressed in the future STCF experiment, for instance, with a new subdetector that can detect the particles emitting from the gaps in ECL, the one order of magnitude increase in sensitivity from Belle II to STCF could be achieved.
We further computed the limits with the optimized detector cuts, shown as dashed curves in Fig. 8. The optimized detector cuts can further enhance the sensitivity of STCF and Belle II in probing the low-mass region.

\section{SUMMARY}

In this paper, we analyzed the sensitivity to millicharged particles from four different electron colliders operated at the GeV scale: BABAR, Belle II, BESIII, and STCF. By reanalyzing the $28 \mathrm{fb}^{-1}$ monophoton data collected by $B A B A R$, one is able to eliminate some currently allowed millicharge parameter space for $\sim(0.5-3.5) \mathrm{GeV}$ mass. The BESIII experiment can probe an even larger region of parameter space than $B A B A R$, owing to the lower colliding energy. The expected limit on MCPs from BESIII is near $\epsilon \sim 10^{-3}$ for $100 \mathrm{MeV}$ mass. Projected limits with Belle II and STCF experiments are also analyzed. It is found that Belle II can probe millicharge down to $\epsilon \sim 10^{-3}-10^{-2}$ for $0.1 \mathrm{GeV} \lesssim m \lesssim 4 \mathrm{GeV}$. The future STCF can further improve the sensitivity to low-mass MCPs than Belle II because it is operated at lower energy. Millicharge $\epsilon \lesssim$ $\mathcal{O}\left(10^{-4}\right)$ for mass from $3 \mathrm{MeV}$ to about $1 \mathrm{GeV}$ can be probed by the future STCF experiment; this excludes some of the parameter space for explaining the $21 \mathrm{~cm}$ anomaly. The sensitivities computed for BESIII and STCF are obtained without taking into account the gap backgrounds. The more accurate limits require full detailed detector simulations, which is beyond the scope of this work.

\section{ACKNOWLEDGMENTS}

We thank Shenjian Chen, Samuel D. McDermott, Van Que Tran, Jingjing $\mathrm{Xu}$, and Lei Zhang for discussions and correspondence. The work is supported in part by the National Natural Science Foundation of China under Grants No. 11775109, No. U1738134, and No. 11805001.

Note added.-Recently, a new limit from ArgoNeuT [53] appeared which rules out most of the parameter space excluded by the $B A B A R$ data.

\section{APPENDIX A: CRYSTAL BALL FUNCTION}

The normalized crystal ball function is given by [56]

$$
f(x, \bar{x}, \sigma)=N \begin{cases}\exp \left(-\frac{(x-\bar{x})^{2}}{2 \sigma^{2}}\right) & \text { for } \frac{x-\bar{x}}{\sigma}>-\alpha, \\ A\left(B-\frac{x-\bar{x}}{\sigma}\right)^{-n} & \text { for } \frac{x-\bar{x}}{\sigma} \leq-\alpha,\end{cases}
$$




$$
\begin{aligned}
A & =\left(\frac{n}{|\alpha|}\right)^{n} \exp \left(-\frac{|\alpha|^{2}}{2}\right), \\
B & =\frac{n}{|\alpha|}-|\alpha|, \\
N & =\frac{1}{\sigma(C+D)}, \\
C & =\frac{n}{|\alpha|} \frac{1}{n-1} \exp \left(-\frac{|\alpha|^{2}}{2}\right), \\
D & =\sqrt{\frac{\pi}{2}}\left(1+\operatorname{erf}\left(\frac{|\alpha|}{\sqrt{2}}\right)\right) .
\end{aligned}
$$

We use $\alpha=0.811$ and $n=1.79$ for BABAR [52].

\section{APPENDIX B: MAXIMUM MONOPHOTON ENERGY IN REDUCIBLE BG}

The maximum energy of the monophoton occurs when both final states $e^{ \pm}$are emitted at the boundary of ECL and are opposite to the photon in the transverse plane. Thus, the energy-momentum conservation in the c.m. frame gives rise to

$$
\begin{gathered}
E_{\gamma}^{m} \sin \theta_{\gamma}-E_{1} \sin \theta_{1}-E_{2} \sin \theta_{2}=0, \\
E_{\gamma}^{m} \cos \theta_{\gamma}+E_{1} \cos \theta_{1}+E_{2} \cos \theta_{2}=0, \\
E_{\gamma}^{m}+E_{1}+E_{2}=\sqrt{s},
\end{gathered}
$$

where $E_{1}$ and $E_{2}$ are the $e^{ \pm}$energies and $\theta_{1}$ and $\theta_{2}$ are the polar angles corresponding to the boundary of the ECL. The solution for $E_{\gamma}^{m}$ from the above equations yields Eq. (2). Note that the monophoton energy cannot exceed $\sqrt{s} / 2$.
[1] C. Q. Geng and R.E. Marshak, Phys. Rev. D 39, 693 (1989).

[2] B. Holdom, Phys. Lett. 166B, 196 (1986).

[3] B. Holdom, Phys. Lett. B 178, 65 (1986).

[4] R. Foot and X. G. He, Phys. Lett. B 267, 509 (1991).

[5] B. Kors and P. Nath, Phys. Lett. B 586, 366 (2004).

[6] K. Cheung and T. C. Yuan, J. High Energy Phys. 03 (2007) 120.

[7] D. Feldman, Z. Liu, and P. Nath, Phys. Rev. D 75, 115001 (2007).

[8] S. Davidson, B. Campbell, and D. C. Bailey, Phys. Rev. D 43, 2314 (1991).

[9] S. Davidson, S. Hannestad, and G. Raffelt, J. High Energy Phys. 05 (2000) 003.

[10] S. Chatrchyan et al. (CMS Collaboration), Phys. Rev. D 87, 092008 (2013).

[11] A. A. Prinz et al., Phys. Rev. Lett. 81, 1175 (1998).

[12] E. Golowich and R. W. Robinett, Phys. Rev. D 35, 391 (1987).

[13] D. E. Soper, M. Spannowsky, C. J. Wallace, and T. M. P. Tait, Phys. Rev. D 90, 115005 (2014).

[14] Z. Liu and Y. Zhang, Phys. Rev. D 99, 015004 (2019).

[15] A. Haas, C. S. Hill, E. Izaguirre, and I. Yavin, Phys. Lett. B 746, 117 (2015).

[16] Z. Liu, Y. H. Xu, and Y. Zhang, J. High Energy Phys. 06 (2019) 009.

[17] S. N. Gninenko, D. V. Kirpichnikov, and N. V. Krasnikov, Phys. Rev. D 100, 035003 (2019).

[18] X. Chu, J. Pradler, and L. Semmelrock, Phys. Rev. D 99, 015040 (2019).

[19] A. Berlin, N. Blinov, G. Krnjaic, P. Schuster, and N. Toro, Phys. Rev. D 99, 075001 (2019).
[20] M. I. Dobroliubov and A. Y. Ignatiev, Phys. Rev. Lett. 65, 679 (1990).

[21] R. N. Mohapatra and I. Z. Rothstein, Phys. Lett. B 247, 593 (1990).

[22] J. H. Chang, R. Essig, and S. D. McDermott, J. High Energy Phys. 09 (2018) 051.

[23] S. L. Dubovsky, D. S. Gorbunov, and G. I. Rubtsov, Pis'ma Zh. Eksp. Teor. Fiz. 79, 3 (2004) [JETP Lett. 79, 1 (2004)].

[24] A. D. Dolgov, S. L. Dubovsky, G. I. Rubtsov, and I. I. Tkachev, Phys. Rev. D 88, 117701 (2013).

[25] S. Davidson and M.E. Peskin, Phys. Rev. D 49, 2114 (1994).

[26] H. Vogel and J. Redondo, J. Cosmol. Astropart. Phys. 02 (2014) 029.

[27] N. Vinyoles and H. Vogel, J. Cosmol. Astropart. Phys. 03 (2016) 002.

[28] S. N. Gninenko, N. V. Krasnikov, and A. Rubbia, Phys. Rev. D 75, 075014 (2007).

[29] G. Magill, R. Plestid, M. Pospelov, and Y. D. Tsai, Phys. Rev. Lett. 122, 071801 (2019).

[30] K. J. Kelly and Y.D. Tsai, Phys. Rev. D 100, 015043 (2019).

[31] L. Singh et al. (TEXONO Collaboration), Phys. Rev. D 99, 032009 (2019).

[32] R. Harnik, Z. Liu, and O. Palamara, J. High Energy Phys. 07 (2019) 170.

[33] J. D. Bowman, A. E. E. Rogers, R. A. Monsalve, T. J. Mozdzen, and N. Mahesh, Nature (London) 555, 67 (2018).

[34] J. B. Muoz and A. Loeb, Nature (London) 557, 684 (2018).

[35] R. Barkana, N. J. Outmezguine, D. Redigolo, and T. Volansky, Phys. Rev. D 98, 103005 (2018). 
[36] A. Berlin, D. Hooper, G. Krnjaic, and S. D. McDermott, Phys. Rev. Lett. 121, 011102 (2018).

[37] E. D. Kovetz, V. Poulin, V. Gluscevic, K. K. Boddy, R. Barkana, and M. Kamionkowski, Phys. Rev. D 98, 103529 (2018).

[38] K. K. Boddy, V. Gluscevic, V. Poulin, E. D. Kovetz, M. Kamionkowski, and R. Barkana, Phys. Rev. D 98, 123506 (2018).

[39] N. Klop and S. Ando, Phys. Rev. D 98, 103004 (2018).

[40] C. Creque-Sarbinowski, L. Ji, E. D. Kovetz, and M. Kamionkowski, Phys. Rev. D 100, 023528 (2019).

[41] H. Liu, N. J. Outmezguine, D. Redigolo, and T. Volansky, Phys. Rev. D 100, 123011 (2019).

[42] E. Kou et al. (Belle II Collaboration), Prog. Theor. Exp. Phys. (2019) 123C01; (2020) 029201(E).

[43] X. Wang, https://indico.ihep.ac.cn/event/9272/session/21/ contribution/49/material/slides/0.pdf.

[44] D. M. Asner et al., Int. J. Mod. Phys. A 24, S1-794 (2009).

[45] H. Peng, https://indico.inp.nsk.su/event/15/session/0/ contribution/99/material/slides/0.pdf.
[46] J. P. Lees et al. (BABAR Collaboration), Nucl. Instrum. Methods Phys. Res., Sect. A 726, 203 (2013).

[47] T. Ferber, https://indico.cern.ch/event/757995/contributions/ 3315335/attachments/1832761/3001940/2019_04_ferber_ ALPS.pdf.

[48] P. f. Yin, J. Liu, and S. h. Zhu, Phys. Lett. B 679, 362 (2009).

[49] http://english.ihep.cas.cn/bes/doc/2250.html.

[50] M. Ablikim et al. (BESIII Collaboration), Phys. Rev. D 96, 112008 (2017).

[51] B. Aubert et al. (BABAR Collaboration), arXiv:0808.0017.

[52] R. Essig, J. Mardon, M. Papucci, T. Volansky, and Y. M. Zhong, J. High Energy Phys. 11 (2013) 167.

[53] R. Acciarri et al. (ArgoNeuT Collaboration), Phys. Rev. Lett. 124, 131801 (2020).

[54] C. Boehm, M. J. Dolan, and C. McCabe, J. Cosmol. Astropart. Phys. 08 (2013) 041.

[55] T. R. Slatyer, Phys. Rev. D 93, 023527 (2016).

[56] L. Pescatore, Searching for new physics in $b \rightarrow s 1+1-$ transitions at the $\mathrm{LHCb}$ experiment, University of Birmingham, Ph.D. thesis. 